\title{
Engineering mesenchymal stem cells with neutralizing and anti- inflammation dual-capability against SARS-CoV-2 infection
}

\section{Xiaoqing Zhang}

Sheng Yushou Center of Cell Biology and Immunology, School of Life Sciences and Biotechnology, Shanghai Jiao Tong University, Shanghai 200240, China

\section{Ping Han}

Sheng Yushou Center of Cell Biology and Immunology, School of Life Sciences and Biotechnology, Shanghai Jiao Tong University, Shanghai 200240, China

\section{Haiyong Wang}

Sheng Yushou Center of Cell Biology and Immunology, School of Life Sciences and Biotechnology, Shanghai Jiao Tong University, Shanghai 200240, China

\section{Yanqin Xu}

Sheng Yushou Center of Cell Biology and Immunology, School of Life Sciences and Biotechnology, Shanghai Jiao Tong University, Shanghai 200240, China

\section{Fanlin Li}

Sheng Yushou Center of Cell Biology and Immunology, School of Life Sciences and Biotechnology, Shanghai Jiao Tong University, Shanghai 200240, China

\section{Min Li}

Sheng Yushou Center of Cell Biology and Immunology, School of Life Sciences and Biotechnology, Shanghai Jiao Tong University, Shanghai 200240, China

\section{Lilv Fan}

Sheng Yushou Center of Cell Biology and Immunology, School of Life Sciences and Biotechnology, Shanghai Jiao Tong University, Shanghai 200240, China

\section{Huihui Zhang}

Sheng Yushou Center of Cell Biology and Immunology, School of Life Sciences and Biotechnology, Shanghai Jiao Tong University, Shanghai 200240, China

\section{Qiang Dai}

Sheng Yushou Center of Cell Biology and Immunology, School of Life Sciences and Biotechnology, Shanghai Jiao Tong University, Shanghai 200240, China

\section{Hao Lin}

Sheng Yushou Center of Cell Biology and Immunology, School of Life Sciences and Biotechnology, Shanghai Jiao Tong University, Shanghai 200240, China

\section{Xinyue Qi}


Sheng Yushou Center of Cell Biology and Immunology, School of Life Sciences and Biotechnology, Shanghai Jiao Tong University, Shanghai 200240, China

\section{Jie Liang}

Sheng Yushou Center of Cell Biology and Immunology, School of Life Sciences and Biotechnology, Shanghai Jiao Tong University, Shanghai 200240, China

\section{Xin Wang}

Shanghai Longyao Biotechnology Limited, Shanghai 201203, China

\section{Xuanming Yang ( $\sim$ xuanmingyang@sjtu.edu.cn )}

Sheng Yushou Center of Cell Biology and Immunology, School of Life Sciences and Biotechnology, Shanghai Jiao Tong University, Shanghai 200240, China

\section{Research Article}

Keywords: SARS-CoV-2, entry, mice, ACE2 extracellular domain-Fc and scFv-IL6R-Fc fusion protein

Posted Date: June 2nd, 2020

DOI: https://doi.org/10.21203/rs.3.rs-32959/v1

License: (c) (i) This work is licensed under a Creative Commons Attribution 4.0 International License. Read Full License 


\section{Abstract}

The emergence of the novel human coronavirus SARS-CoV-2 has caused a worldwide epidemic of coronavirus disease 2019 (COVID-19), which markedly affected the global health and economy. Both uncontrolled viral replication and proinflammatory cytokine storm can cause severe tissue damage in COVID-19 patients. SARS-CoV-2 utilizes angiotensin-converting enzyme 2 (ACE2) as its entry receptor. In this study, we generated ACE2 extracellular domain-Fc and scFv-IL6R-Fc fusion protein to differentially neutralize virus and temper cytokine storm. The hACE21-740-Fc fusion protein showed potent inhibitory effect on pseudotyped SARS-CoV-2 entry and good safety profile in mice. The scFv-IL6R-Fc showed strong blockade effect on IL-6 signal activation. In addition, we established a mesenchymal stem cells (MSCs)-based hACE21-740-Fc and scFv-IL6R-Fc delivery strategy, which provided a potential rapid option for urgent clinic therapeutic need of COVID-19 patients.

\section{Introduction}

Emerging infectious diseases present a huge challenge to human health and have great influence on global stability, such as SARS-CoV in 2003 and MERS-CoV in 20121-3. In December 2019, a novel coronavirus (SARS-CoV-2) was reported to infect human and cause an outbreak of coronavirus disease 2019 (COVID-19) in Wuhan, China4,5. The symptoms of COVID-19 include asymptomatic infection, mild disease, acute lung injury (ALI), multiple organ failure (MOF), and even death6. So far, this disease has rapidly spread from Wuhan to all over the world. The World Health Organization declared COVID-19 as a pandemic on March 14th, 2020. Until May 2020, there are more than four million confirmed SARSCoV-2 infections, placing a tremendous burden to public health and global economy.

SARS-CoV-2 belongs to the Betacoronavirus genera and was closer to SARS-like coronavirus in bat. Phylogenetic analysis revealed that it is approximately $80 \%$ similar to SARS-CoV in the genome sequence. Specifically, these two viruses exhibit $74 \%$ amino acid sequence similarity in the receptor binding domain (RBD) of spike gene, suggesting a possible common host receptor. Indeed, angiotensin converting enzyme 2 (ACE2) has been recognized as the critical entry receptor of both SARS-CoV-2 and SARS-CoV 7,8. Compared to the spike of SARS-CoV, the higher binding affinity of SARS-CoV-2 spike to hACE2 may contribute to the higher transmission rate of SARS- CoV-29,10, implying the importance of ACE2-dependent viral entry into cells. ACE2 is primarily expressed in lung alveolar epithelial type II cells, which are crucial for the gas exchange function of lung11,12. Severe lung injure in some COVID-19 patients may stem from dysfunction of these cells13 ACE2 is also expressed in multiple other organs, such as heart, liver, intestine, testis, kidney and blood vessels14-18, which may give rise to the multiple organ dysfunction syndrome (MODS) induced by SARS-CoV-2 infection6. Apart from being the entry receptor of SARS-CoV, ACE2 was shown to facilitate tissue repair after lung injury19. Most importantly, soluble recombinant ACE2 protein has exhibited therapeutic potential for the SARS-CoV20.

About $5 \%$ of patients infected with SARS-CoV-2 have developed a sever lung injury symptom, which is accompanied with overwhelmed immune activation. Some anti- virus and inflammation blocking drugs 
have been tested in clinic for treating COVID-1921. However, these single targeting drugs showed minimal clinical effect. Drugs targeting both virus itself and tissue damage are urgently needed for treating severe COVID-19.

The multi-potent differential potential, the tissue repair ability and the anti- inflammatory property of MSCs potentiate their clinical development for the treatment of autoimmune disease22,23. Besides, MSCs exert the paracrine functions through secreting soluble factors and releasing extracellular vesicles, and possess the innate ability to traffic to the inflammation sites, which made them a promising drug delivery vector in preclinical models24. Here we represent a MSC-based cell therapy to simultaneously block SARS-CoV-2 entry and inhibit virus induced cytokine storm. This dual targeting strategy provided a rapid clinic-ready, multiple layers of resolution to COVID-19 therapy.

\section{Results}

\section{ACE2-Fc bound to SARS-CoV-2 spike with high affinity}

It is reported that SARS-CoV-2 enters into permissive cells through ACE27,25 Competitive soluble extracellular domains of ACE2 thus might be potential viral entry blocker. To test whether ACE2 extracellular domain could block SARS-CoV-2 entry, two recombinant ACE2-Fc fusion proteins were generated, consisting of the amino acid 1-615 or 1-740 of ACE2 fused with human IgG1 Fc portion respectively. We then characterized their binding profile with SARS-CoV-2 spike protein. By the similar strategy, another two recombinant SARS-CoV-2 spike-Fc fusion proteins were generated as well, containing amino acid 1-685(S1) or amino acid 319-541(RBD) fused to mouse IgG2a Fc portion (Figure 1A). Through an ELISA binding assay, both ACE21-615-hFc and ACE21-740-hFc bound to S1-mFc and RBD-mFc with similar affinity (Figure 1C\&D). These data suggested that $\mathrm{S} 1$ and RBD region are essential for the binding of ACE2 and SARS-CoV-2 spike. To test the binding ability of ACE2-hFc fusion protein to SARS-CoV-2 spike in a more physiological condition, we established the 293-spike cell line, which stably expressed SARS-CoV-2 spike protein. By flow cytometry analysis, both ACE21-615-hFc and ACE21-740hFc bound to 293-spike with similar affinity (Figure 1E). These data collectively suggested that the extracellular domain of ACE2-Fc fusion protein can bind to SARS-CoV-2 spike protein with high affinity and could be used as a potential entry blocker.

\section{ACE2-Fc blocked the entry of pseudotyped SARS-CoV-2 virus into ACE2+ cells}

Due to the potential risk of severe COVID-19 induced by SARS-CoV-2 infection, researches on SARSCoV-2 must be conducted in laboratories with a biosafety level (BSL) of 3 or higher, which becomes a hurdle to SARS-CoV-2 research. To overcome this limitation, we have established a pseudotyped SARSCoV-2 virus system, which replaced the VSV-G with SARS-CoV-2 spike to produce a SARS-CoV-2 pseudotyped virus utilizing the well-defined 3rd generation of lentivirus system (Figure 2A). We first 
checked the infection ability of SARS-CoV-2 pseudotyped virus in both ACE2+ and ACE2-293 cells. SARS-CoV-2 pseudotyped virus can only infect ACE2+ 293 cells, rather than ACE- 293 cells, suggesting a SARS-CoV-2 spike-mediated, ACE2- dependent entry process (Figure 2B). By using this pseudotyped virus system, we next tested the blocking efficacy of ACE21-615-Fc and ACE21-740-Fc. Indeed, ACE21740-Fc showed a strong blockade effect by inhibiting the entry of SARS-CoV2 pseudotyped virus into 293ACE2 cell with an IC50 of $\sim 0.68 \mathrm{nM}$ (Figure 2C). To our surprise, the ACE21-615-Fc showed very low blocking effect on the entry of SARS-CoV-2 pseudotyped virus despite its similar binding profile to S1mFc, RBD-mFc and 293- spike cells (Figure 2D \& Figure 1C-E). To further test the specificity of ACE21740-hFc- mediated blockade of pseudotyped SARS-CoV-2 virus, we investigated the blocking effect of ACE21-740-hFc on the entry of VSV pseudotyped virus. Although ACE21-740- Fc inhibited 80\% infection of SARS-CoV-2 pseudotyped virus, it had no effect on the infection of VSV pseudotyped virus (Figure 2E). To test whether ACE21-740-Fc - mediated blockade could be applicable to other types of cell, we performed similar assay on lung epithelial A549-ACE2 cell line. Consistent with the data from 293ACE2 cells, ACE21-740-Fc significantly blocked the entry of SARS-CoV-2 pseudotyped virus into A549ACE2 cells (Figure 2F). Collectively, ACE21-740-hFc could block pseudotyped SARS-CoV-2 virus infection through competing with membrane- anchored entry receptor.

\section{ACE2-Fc mediated cytotoxicity on SARS-CoV-2 spike+ cells through Fc portion.}

There are two functional portions in the ACE21-740-hFc fusion protein. The extracellular domain of ACE21-740 portion functions as a competitive binder to membrane-anchored SARS-CoV-2 receptor ACE2, while the Fc portion can mediate multiple functions through engaging FcyR positive cells and complements ${ }^{26}$. To verify whether ACE21-740-hFc could induce cell death of SARS-CoV-2 spike positive cells through Antibody- dependent Cell-mediated Cytotoxicity (ADCC) effect, we performed a NK cell and

Raji-spike cell co-culture assay in the presence of ACE21-740-hFc. Due to the high binding affinity to activating FcyRs of IgG127, ACE21-740-hFc induced a significant ADCC effect and lysed more Raji-spike cells than Raji cells (Figure $3 A$ ), suggesting a potential lysis effect on virus-infected cells in vivo. We also tested whether ACE21-740- hFc could induce Complement-Dependent Cytotoxicity (CDC) effect. When complements were present, ACE21-740-hFc induced more lysis of spike positive cells than spike negative cells (Figure 3B). Taken together, ACE21-740-hFc could potentially eliminate virus-infected spike+ cells through both ADCC and CDC effect.

\section{Safety profile of ACE21-740-hFc in vivo}

ACE2 is a carboxypeptidase that potently degrades angiotensin II to angiotensin 1-728. ACE2 antagonizes the activation of the classical RAS system and protects against organ damage, hypertension, diabetes, and cardiovascular disease29. However, whether exogenous exposure of ACE21- 
740-hFc could affect the normal physiology status is not clear. Firstly, we tested the in vivo half-life of hACE21-740-hFc in mice. The half-life of intact hACE21-740-hFc in vivo is about 8.22 hours (Figure 4A). Subsequently, different tissues were collected to investigate the potential effect of ACE21-740-hFc infusion on tissue damage. There were no difference in hematological feature, body weight change, ALT and immune cell infiltration in the organs of heart, liver, spleen, lung, and kidney (Figure 4B-E). Although the enzyme activity of hACE21-740-hFc functions well in mice30, two potential factors may affect the evaluation of side effects, namely, potential immune response against human protein hACE2 in mice and different interaction network of human ACE2 compared with mouse ACE2. To avoid these limitations, we performed similar experiments by using mACE21-740-hFc and did not observe adverse effects in seven days after treatment (Figure 4B-E). Collectively, ACE21-740-hFc was well tolerated in mice without obvious side effect.

\section{Engineered MSCs as a drug development strategy for urgent need of COVID-19 treatment}

It is time consuming for protein-based therapeutics to enter the clinic due to good manufacturing practices (GMP) production, and quality control, which cannot meet the urgent need for treating SARSCoV-2 induced COVID-19. To this end, other drug delivery systems should be considered to save the time on development. Mesenchymal stem cells have been widely used for treating autoimmune disease and as the vector for protein drug delivery, which could be a good option. We then generated the MSC cell line stably expressing ACE21-740-hFc (MSC-ACE21-740-hFc) by lentiviral infection. As expected, this cell line secreted ACE21-740-hFc into supernatant persistently (Figure 5A). The concentration of ACE21$740-\mathrm{hFc}$ in supernatant is about $190 \mathrm{ng} / \mathrm{ml}$ after a 4-day cell culture. To recognize whether ACE21-740$\mathrm{hFc}$ in MSC-derived supernatant is bio- reactive, we first measured its binding ability to spike positive 293spike cells. Actually, the MSC-secreted ACE21-740-hFc showed similar binding strength to the purified ACE21-740-hFc (Figure 5B). Since it has the equivalent binding ability, we next sought to identify whether ACE21-740-hFc in MSC-derived supernatant could block pseudotyped virus entry. Indeed, the MSCderived supernatants showed significant blockade effect on the entry of pseudotyped SARS-CoV-2 virus into 293-ACE2 cells (Figure 5C). To test the possibility of using MSC-ACE2-1-740-hFc as a live drug in vivo, we then injected these engineered MSCs into mice. Interestingly, the expression of ACE2-Fc in serum lasted for more than 14 days after one single infusion $\otimes$ Figure 5D囚. Most importantly, when we checked its bio-distribution, the MSC preferentially located in lung at 24 hours after injection区Figure 5E), suggesting a potential usage for treating SARS-CoV-2-induced pneumonia. Therefore, MSC-based ACE21-740-Fc delivery strategy provided a time-saving option for treating clinical SARS-CoV-2-induced disease.

\section{MSC expressing scFv-IL6R-Fc as a potential strategy to inhibit SARS-CoV-2-induced immune damage}


Proinflammatory cytokine storm can cause tissue damage in multiple disease models. Blocking of proinflammatory cytokine such as IL -6 has shown therapeutic effect in autoimmune disease and CAR-T cell-induced cytokine storm31,32. For example, Tocilizumab has been used for treating COVID-19 and improved patient outcome33,34. Considering that MSC is capable of producing proteins rapidly and preferentially migrating to inflammatory tissues, we aim to generate ScFV-IL6R-Fc expressing MSC as a treatment option for SARS-CoV-2-induced cytokine storm. To accelerate this process, we first generated ScFv-IL6R-Fc protein. We purified it and compared its activity with Tocilizumab. The scFv-IL6R-Fc showed similar blockade effect on IL-6- induced TF-1 cell proliferation (Figure 6A). We then established the MSC cell line stably expressing scFv-IL-6R-Fc. After one-month culture, this cell line was still capable of secreting scFv-IL6R-Fc. The concentration of scFv-IL6R- Fc in supernatant was about $300 \mathrm{ng} / \mathrm{ml}$ after a 4day cell culture (Figure 6B). To test the persistence of MSC-derived ScFv-IL6R-Fc in vivo, we then injected these engineered scFv-IL6R-Fc expressing cell into mice. Interestingly, the expression of ACE2-Fc in serum lasted for more than 14 days after one single infusion $\otimes F i g u r e 6 \mathrm{C} \otimes$. Therefore, the scFv-IL-6R- Fcexpressing MSCs can be potentially used for blocking SARS-CoV-2-induced cytokine storm, which can be combined with viral neutralizing MSC-ACE21-740-Fc to provide multiple layers of protection against SARS-CoV-2 infection.

\section{Discussion}

As the most crucial global health crisis of the century, the ongoing COVID-19 pandemic has created a much greater social and economic impact than either of its predecessors, SARS outbreak and MERS epidemic. To date, no clinically approved antiviral drugs or vaccines have been proven effective against COVID-19. Although some drugs showed in vitro anti-SARS-CoV activity, the clinical efficacy of these drugs are dismal. A safe and effective treatment for COVID-19 is urgently needed. SARS-CoV-2 enters into the permissive cells mainly through ACE27. Both neutralizing antibodies against SARS-CoV-2 spike and soluble ACE2 receptors are potential drug candidates with the ability to block viral entry 13,35 .The good profile of stability, specificity and manufacture process has made neutralizing antibody a promising candidate for drug development. Theoretically, it only affects virus or virus-infected cells and thus will have limited side effect in vivo. However, with the high mutation rate, virus may escape neutralizing antibody via single mutations. It is supported by the observation that neutralizing antibodies against SARS-CoV have limited blocking effect on SARS-CoV-2, although they are highly similar in the structure of RBD domain. However, there is no such trouble in the soluble ACE2-based neutralization strategy, since mutant virus escaping soluble ACE2 will also lose the binding ability to ACE2 positive cells. There is a limitation for both neutralizing antibody and ACE2-Fc fusion protein: antibody dependent enhancement effect to virus infection. Further experiments need to be performed to clarify these concerns. Due to biosafety issue, our experiment was performed with pseudotyped virus. Although the entry step of pseudotyped virus is similar to live virus, further investigation with live virus and animal model will provide more information on the efficacy of ACE2-Fc based therapy.

ACE2 is a carboxypeptidase that potently degrades angiotensin II to angiotensin 1-728. It plays a central role in the homeostatic control of cardiorenal actions. ACE2 is a membrane protein, its distribution is 
determined by the expressing cells. While soluble ACE2 will deliver to all possible tissue through system circulation. This raised the concern that whether soluble ACE2 has potential side effect to the cardiorenal system. Our preliminary mouse data showed that there is no obvious side effect being induced after ACE2-Fc treatment. Further investigation on the long-term side effect of ACE2 needs to be conducted on animal models before clinical study. Despite this limitation, there is one potential advantage of soluble ACE2 as a promising therapy. ACE2 was reported to play protective role in lung injury19. SARS-CoV-2 infection induces internalization of ACE2 receptor, which may reduce the ACE2 enzymatic activity and the protective effect on lung injury. Supplement of soluble ACE2 may provide additional tissue repair effect beyond viral neutralization.

To meet the urgent need for treating SARS-CoV-2 induced COVID-19, we have developed a MSC-based cell therapy strategy. MSC has been widely used for treating autoimmune diseases for more than twenty years in clinic36. There are some ongoing clinical trials using MSC to treat COVID-19 due to its innate anti-inflammation and tissue repair ability. Now we armed it with virus neutralization and anti-cytokine storm capability, which will significantly boost its therapeutic effect. MSCs preferentially locate in inflammation tissue, providing a targeted delivery of ACE-2 fusion proteins to SARS-CoV-2 infected tissue. This will significantly enhance the drug concentration in targeted tissue and lower the potential side effect. However, there is a potential disadvantage for MSC-based therapy that it is challenging to regulate the dosage of soluble ACE2 secreted from cells. Further engineering of MSC with on-off switchable promoter or suicide gene will provide possible solution to overcome these hurdles.

Collectively, our data indicated that ACE2-Fc has the potential to be a therapeutic drug for treating COVID -19 with a relatively good safety profile. MSC-based viral blockade and inflammation control provided a rapid option for multiple layers of protection against SARS-CoV-2 infection.

\section{Materials And Methods}

\section{Mice}

C57BL/6J mice were purchased from Beijing Vital River Laboratory Animal Technology Co., Ltd. (Beijing, China). NOD-PrkdcscidIL2rytm1 (NSG) mice were purchased from Shanghai Model Organisms Center, Inc. (Shanghai, China). All mice were maintained under specific pathogen-free conditions. Animal care and use were in accordance with institutional and $\mathrm{NIH}$ protocols and guidelines, and all studies were approved by the Animal Care and Use Committee of Shanghai Jiao Tong University. Cell lines and reagents

Lenti-X 293 was purchased from Clontech. A549 cell line was obtained from the American Type Culture Collection. Raji cells were kindly provided by Stem Cell Bank, Chinese Academy of Sciences (Shanghai, China). TF-1 cells were kindly provided by the Cell Resource Center, Peking Union Medical College (Beijing, China). Human NK cells were kindly provided by Shanghai Longyao Biotechnology Co., Ltd. (Shanghai, China). Plasmids encoding SARS-CoV-2 spike and ACE2 were obtained from MolecularCloud 
(Nanjing, China) and sub-cloned to PCDH-EF (System Biosciences) lentiviral vector plasmid with puromycin resistance marker. To establish SARS-CoV-2 spike or ACE2 expressing cell lines, Lenti-X 293, A549 or Raji were infected with SARS-CoV-2 spike or ACE2- expressing lentivirus. After selection with puromycin, pooled resistant cells were identified by flow cytometry analysis. Cell culture medium were supplemented with $10 \%$ heat-inactivated fetal bovine serum, $2 \mathrm{mmol} / \mathrm{L}$ L- glutamine, $100 \mathrm{units} / \mathrm{mL}$ penicillin, and $100 \mu \mathrm{g} / \mathrm{mL}$ streptomycin. Lenti-X 293, A549 and their derivatives were cultured with complete DMEM medium. Raji and its derivatives were cultured with complete RPMI medium. Human AB serum were purchased from Gemini Bio-products (West Sacramento, CA).

\section{Production of ACE2 and spike fusion protein}

For hACE21-740-hFc hACE21-615-hFc, mACE21-740-hFc, S1-mFc and RBD-mFc fusion protein, DNA sequences encoding the indicated protein (supplemental figure 1) were cloned into $\mathrm{PCDH}-\mathrm{EF}$ vector (System Biosciences, Mountain View, CA). The plasmids containing indicated fusion protein were transfected into Lenti-X 293 cells and supernatants were collected and purified by Diamond Protein A column according to the manufacturer's protocol (Bestchrom, Shanghai, China).

\section{Neutralization assay with pseudotyped SARS-CoV-2 virus}

Lenti-X 293 cells were transfected with lentivirus package component plasmids, Gap/pol (Addgene \#12251, RSV-Rev (Addgene \#12253), pCDH-EF-IRFP-Iuc and pcDNA3.1(+)-2019-nCoV-spike-P2A-eGFP (MolecularCloud, \#MC_0101087). Supernatants containing lentivirus particles were collected 48 and $72 \mathrm{~h}$ post- transfection for direct usage or concentration by ultracentrifugation. Viral titer in $\mathrm{TU} / \mathrm{mL}$ was determined by flow cytometry analysis of transduced Lenti-X 293-ACE2 cells. In the viral neutralization assay, hACE21-740-hFc and hACE21-615-hFc were serially diluted at indicated concentration in complete DMEM medium. Diluted hACE21-740-hFc and hACE21-615-hFc were incubated with pseudotyped lentiviral particles for 15 minutes at room temperature, inoculated on 293-ACE2 or A549ACE2 monolayers in $96-$ well plate in the presence of $10 \mathrm{ug} / \mathrm{ml}$ of polybrene, and further incubated at $37{ }^{\circ} \mathrm{C}$ for 48hours. For infecting A549 -ACE2, a 60-minutes spinning at $2500 \mathrm{RPM}$ at $32^{\circ} \mathrm{C}$ was used to improve the infection efficiency. IRFP reporter activity was measured on a Cytoflex S (Beckman Coulter). The percentage of infectivity was calculated as ratio of IRFP readout in the presence of fusion protein normalized to IRFP readout in the absence of fusion protein. The half maximal inhibitory concentrations (IC50) were determined using 4-parameter logistic regression (GraphPad Prism version 8).

\section{ELISA and Flow cytometry analysis of hACE21-740-hFc and hACE21-615-hFc}

ELISA plates (Jet Biofil, Guangzhou, China) were coated with $2 \mu \mathrm{g} / \mathrm{ml}$ of RBD-mFc at $4{ }^{\circ} \mathrm{C}$ overnight. Plates were washed three times with PBS c ontaining $0.05 \%$ Tween -20 and blocked with $2 \%$ fetal bovine 
serum in PBS at room temperature for 1 hour. Indicated diluted hACE21-740-hFc and hACE21-615-hFc containing samples were added and plates were incubated for 1 hour at room temperature. Plates were washed three times and incubated with alkaline phosphatase (AP)-conjugated goat anti-human Fc secondary antibody (Jackson Immuno. Research) diluted 1:2000 in blocking buffer for 1 hour at room temperature. AP activity was measured at 405 nanometer using p-Nitrophenyl Phosphate (Guangzhou Howei Pharmaceutical Technology Co. Ltd $₫$ Guangzhou, China) by an ELISA plate reader (Molecular Devices). Half-maximum effective concentration (EC50) binding values were calculated by GraphPad Prism (version 8).

293-spike cells were incubated with indicated ACE21-740-hFc and ACE21-615-hFc containing samples at $4{ }^{\circ} \mathrm{C}$ for 30 minutes, washed for three times with $2 \%$ FBS in PBS, incubated with 1:200 diluted Alexa Fluor 647 conjugated goat anti-human Fc antibodies (Jackson Immuno. Research). Samples were analyzed on a Cytoflex S (Beckman Coulter), and data were analyzed with FlowJo software (TreeStar, Inc.).

\section{Lentivirus production}

Lentivirus was produced by transient transfection of Lenti-X 293 with a four plasmid system. Supernatants containing lentivirus particles were collected 48 and $72 \mathrm{~h}$ post- transfection and used for establishing stable cell lines.

\section{In vitro $A D C C$ and $C D C$ assay}

Raji and Raji-spike cells were labeled with 5 or $50 \mu \mathrm{M}$ of CFSE, respectively according to manufacturer's protocol (MedChemExpress, Shanghai, China). Approximately 2x104 CFSE labeled Raji and Raji-spike cells were co-cultured with $4 \times 105$ primary NK cells or supplemented with human AB serum in the presence of various concentration of hACE21-740-hFc. Forty-eight hours later, cell were analyzed by flow cytometry. The ratio of CFSEhigh/CFSElow was used to indicate the relative killing to Raji-spike than Raji.

\section{In vivo characterization of hACE21-740-hFc}

Pharmacokinetics were determined in C57BL/ 6 mice after a single injection of $100 \mu \mathrm{g}$ of ACE21-740$\mathrm{hFc}$. Serum concentrations at indicated time points were determined by ELISA with immobilized RBD$\mathrm{mFc}$. C57BL/6 mice received a single injection of $100 \mu \mathrm{g}$ ACE21-740-hFc. Seven days later, the hematology, body weight, and H\&E staining of indicated organ were analyzed. H\&E staining was performed by Servicebio (Wuhan, China).

\section{Bio-distribution of MSC-ACE2-Fc}


NSG mice were injected with 5-7.5x105 MSC-ACE2-Fc intravenously through retro orbital sinus. Twentyfour hours later, heart, liver, spleen, lung and kidney organs were collected. Genomic DNA was isolated from these organs using an E. Z. N. A. B Tissue DNA Kit I (Omega Bio-tek). The relative distribution of human MSC was quantified by real-time PCR with primers (5'-aagtcataagtcggttgaggggagat-3' and 5'ccagattctgcagttttagcatctgtgt $-3^{\prime}$ ) against human $I L 2 R G$ gene, which was deficient in NSG mice.

\section{TF-1 proliferation assay}

TF-1 cells were plated in flat bottomed 96 -well plates at 10,000 cells/well in the presence of 1,10 or $50 \mathrm{ng} / \mathrm{ml}$ of IL-6 (Sino Biological, Beijing, China). Then $0.1,1$ or $10 \mu \mathrm{g} / \mathrm{ml}$ of scFv-IL6R-Fc or Tocilizumab (Roche) were added to the cell culture. Forty eight hours later, Cell proliferation was measured by CCK- 8 assay (Dojindo Molecular Technologies, Shanghai, China) according to manufacturer's protocol.

\section{Statistical analysis}

Data are expressed as means \pm standard deviation (SD) or standard error of the mean (SEM). The data were compared using two-tailed unpaired Student's $t$ test compared with two groups. Statistically significant differences $p<0.05, p<0.01$, and $p<0.001$ are noted with *, **, and $* \star *$ respectively.

\section{Declarations}

Acknowledgments

We thank Dr. Jie Zhao for comments on and editing of this paper. X.Y. was supported by the Sheng Yushou foundation, National Natural Science Foundation of China (81671643 and 81971467), The National Key Research and Development Program of China (2016YFC1303400) and the Recruitment Program of Global Experts (People's Republic of China). P. H. was supported by National Natural Science Foundation of China (81901689).

Author contributions

X.Y. designed the overall project. X.Z, P.H., H.W., Y. X., M.L., F.L., H.Z., Q.D., H.L.,X.Q., J.L, X.W. and X.Y. performed the experiments. X.Z, and X.Y. analyzed the results and wrote the manuscript.

Competing Interests

The authors declare no competing interests.

Data availability

All relevant data are available from the authors on request. Supporting data of this study are available from the corresponding author on reasonable request. Source data for graphs and tables presented in 
this manuscript are provided as a Source Data file.

\section{Reference}

1. Drosten, , et al. Identification of a novel coronavirus in patients with severe acute respiratory syndrome. N Engl J Med 348, 1967-1976 (2003).

2. Ksiazek, G., et al. A novel coronavirus associated with severe acute respiratory syndrome. N Engl J Med 348, 1953-1966 (2003).

3. Zaki, A.M., van Boheemen, S., Bestebroer, M., Osterhaus, A.D. \& Fouchier, R.A. Isolation of a novel coronavirus from a man with pneumonia in Saudi Arabia. N Engl J Med 367, 1814-1820 (2012).

4. Ren, L.L., et al. Identification of a novel coronavirus causing severe pneumonia in human: a descriptive Chin Med J (Engl) 133, 1015-1024 (2020).

5. Zhu, , et al. A Novel Coronavirus from Patients with Pneumonia in China, 2019. N Engl J Med 382, 727-733 (2020).

6. Huang, C., et al. Clinical features of patients infected with 2019 novel coronavirus in Wuhan, China. Lancet 395, 497-506 (2020).

7. Zhou, , et al. A pneumonia outbreak associated with a new coronavirus of probable bat origin. Nature 579, 270-273 (2020).

8. Li, H., et al. Angiotensin-converting enzyme 2 is a functional receptor for the SARS coronavirus. Nature 426, 450-454 (2003).

9. Wang, , et al. Structural and Functional Basis of SARS-CoV-2 Entry by Using Human ACE2. Cell 181, 1-11 (2020).

10. Wrapp, , Wang, N., Corbett, K.S., Goldsmith, J.A., Hsieh, C.L., Abiona, O., \& Graham, B.S., and McLellan, J.S. Cryo-EM structure of the 2019-nCoV spike in the prefusion conformation. science 367, 12601263 (2020).

11. Zhao, , et al. Single-cell RNA expression profifiling of ACE2, the putative receptor of Wuhan 2019nCov. (2020).

12. Dobbs, L.G. Pulmonary surfactant. Annu Rev Med 40, 431-446 (1989).

13. Monteil, , et al. Inhibition of SARS-CoV-2 Infections in Engineered Human Tissues Using ClinicalGrade Soluble Human ACE2. Cell 181, 1-9 (2020).

14. Crackower, A., et al. Angiotensin-converting enzyme 2 is an essential regulator of heart function. Nature 417, 822-828 (2002).

15. Danilczyk, U. \& Penninger, M. Angiotensin-converting enzyme II in the heart and the kidney. Circ Res 98, 463-471 (2006).

16. Guan, J., et al. Clinical Characteristics of Coronavirus Disease 2019 in China. The New England journal of medicine 382, 1708-1720 (2020). 
17. Gu, , et al. Multiple organ infection and the pathogenesis of SARS. The Journal of experimental medicine 202, 415-424 (2005).

18. Hamming, l., et al. Tissue distribution of ACE2 protein, the functional receptor for SARS coronavirus. A first step in understanding SARS J Patho/ 203, 631-637 (2004).

19. Zhang, H.B., Penninger, M., Li, Y.M., Zhong, N.S. \& Slutsky, A.S. Angiotensin-converting enzyme 2 (ACE2) as a SARS-CoV-2 receptor: molecular mechanisms and potential therapeutic target. Intens Care Med 46, 586-590 (2020).

20. Kuba, K., et al. A crucial role of angiotensin converting enzyme 2 (ACE2) in SARS coronavirusinduced lung injury. Nat Med 11, 875-879 (2005).

21. Lythgoe, P. \& Middleton, P. Ongoing Clinical Trials for the Management of the COVID-19 Pandemic. Trends Pharmacol Sci (2020).

22. Le Blanc, K., et al. Mesenchymal stem cells for treatment of steroid-resistant, severe, acute graftversus-host disease: a phase II Lancet 371, 1579-1586 (2008).

23. Duijvestein, M., et al. Autologous bone marrow-derived mesenchymal stromal cell treatment for refractory luminal Crohn's disease: results of a phase I Gut 59, 1662-1669 (2010).

24. Ranganath, S.H., Levy, , Inamdar, M.S. \& Karp, J.M. Harnessing the mesenchymal stem cell secretome for the treatment of cardiovascular disease. Cell Stem Cell 10, 244-258 (2012).

25. Hoffmann, M., et al. SARS-CoV-2 Cell Entry Depends on ACE2 and TMPRSS2 and Is Blocked by a Clinically Proven Protease Inhibitor. Cell 181, 271-280 e278 (2020).

26. Nimmerjahn, \& Ravetch, J.V. Fcgamma receptors as regulators of immune responses. Nat Rev Immunol 8, 34-47 (2008).

27. Kiyoshi, , et al. Structural basis for binding of human IgG1 to its high-affinity human receptor FcgammaRI. Nat Commun 6, 6866 (2015).

28. Hamming, l., et al. The emerging role of ACE2 in physiology and disease. J Patho/ 212, 1-11 (2007).

29. Jiang, , et al. Angiotensin-converting enzyme 2 and angiotensin 1-7: novel therapeutic targets. Nat Rev Cardiol 11, 413-426 (2014).

30. Wysocki, J., Schulze, A. \& Batlle, Novel Variants of Angiotensin Converting Enzyme-2 of Shorter Molecular Size to Target the Kidney Renin Angiotensin System. Biomolecules 9(2019).

31. Yao, , et al. Targeting interleukin-6 in inflammatory autoimmune diseases and cancers. Pharmacol Ther 141, 125-139 (2014).

32. Lee, W., et al. Current concepts in the diagnosis and management of cytokine release syndrome. Blood 124, 188-195 (2014).

33. Xu, X., et al. Effective treatment of severe COVID-19 patients with tocilizumab. Proc Natl Acad Sci U S $A(2020)$.

34. Toniati, , et al. Tocilizumab for the treatment of severe COVID-19 pneumonia with hyperinflammatory syndrome and acute respiratory failure: A single center study of 100 patients in Brescia, Italy. Autoimmun Rev, 102568 (2020). 
35. Lei, , et al. Neutralization of SARS-CoV-2 spike pseudotyped virus by recombinant ACE2-lg. Nat Commun 11, 2070 (2020).

36. Frenette, S., Pinho, S., Lucas, D. \& Scheiermann, C. Mesenchymal stem cell: keystone of the hematopoietic stem cell niche and a stepping-stone for regenerative medicine. Annu Rev Immunol 31, 285-316 (2013).

\section{Figures}

Figure 1. ACE2-Fc bound with SARS-CoV-2 spike protein with high affinity

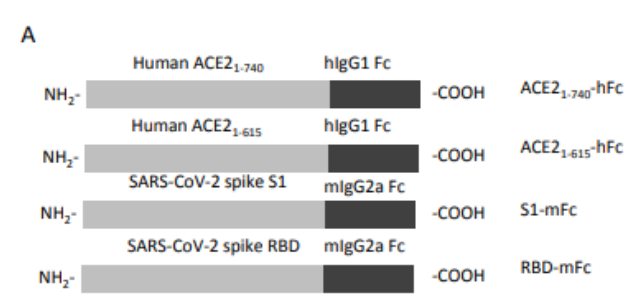

B
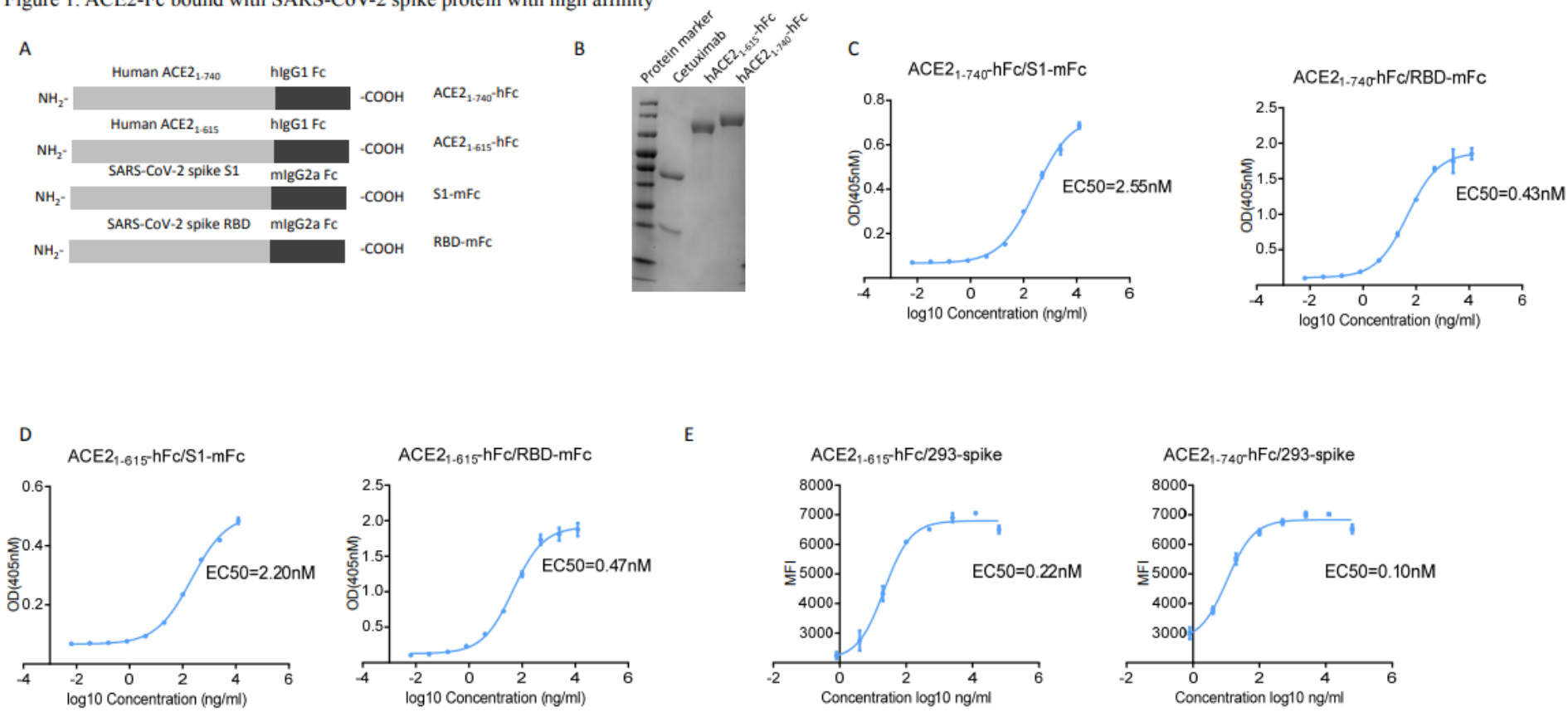

$E$
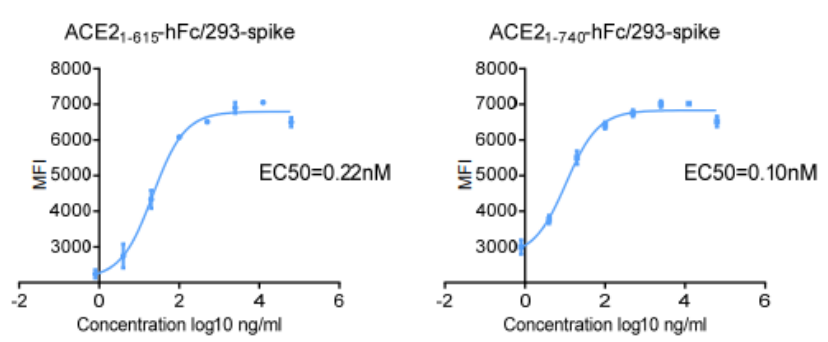

Figure 1

ACE2-Fc bound with SARS-CoV-2 spike protein with high affinity 
A

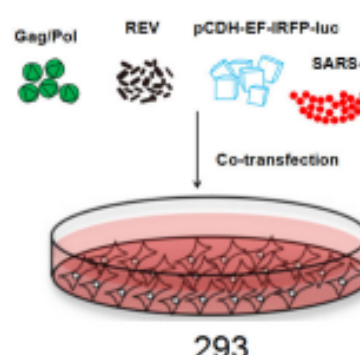

293

C

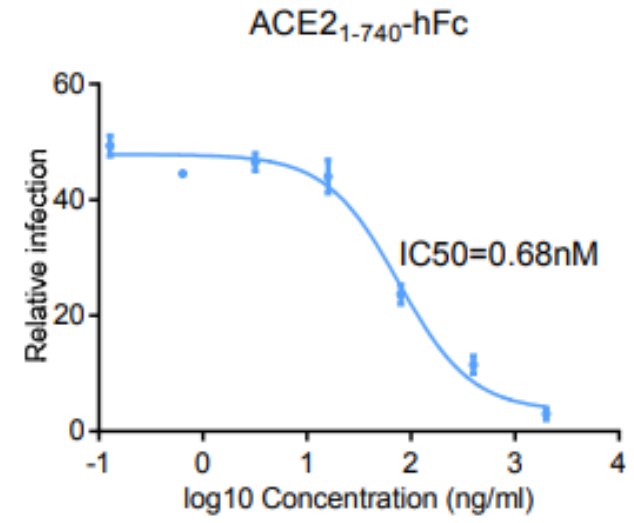

B

293

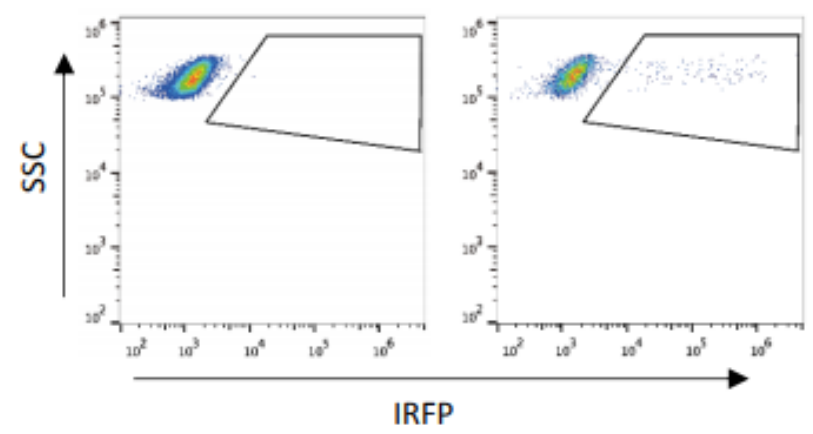

D ACE2 $1-615-\mathrm{hFC}$

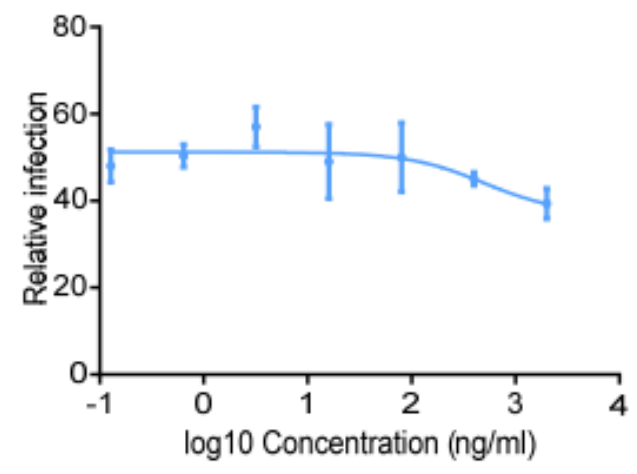

E

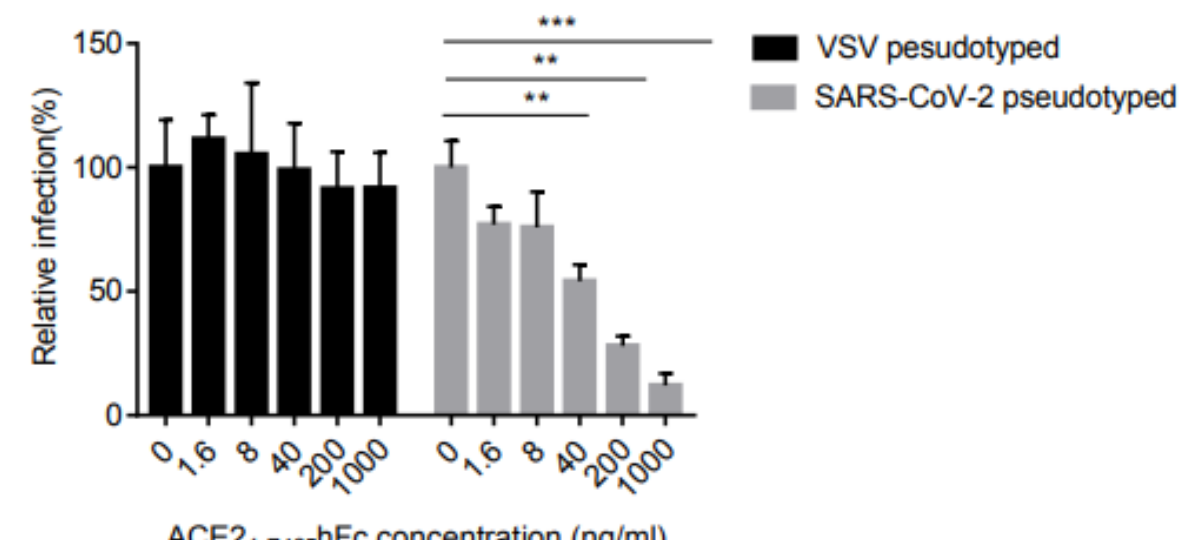

$\mathrm{F}$

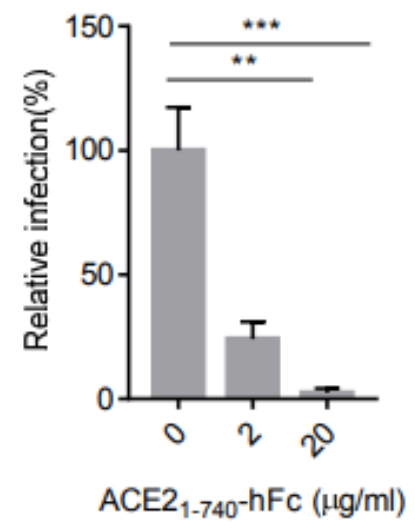

Figure 2

ACE21-740-hFc blocked pseudotyped SARS-CoV-2 virus infection 
A
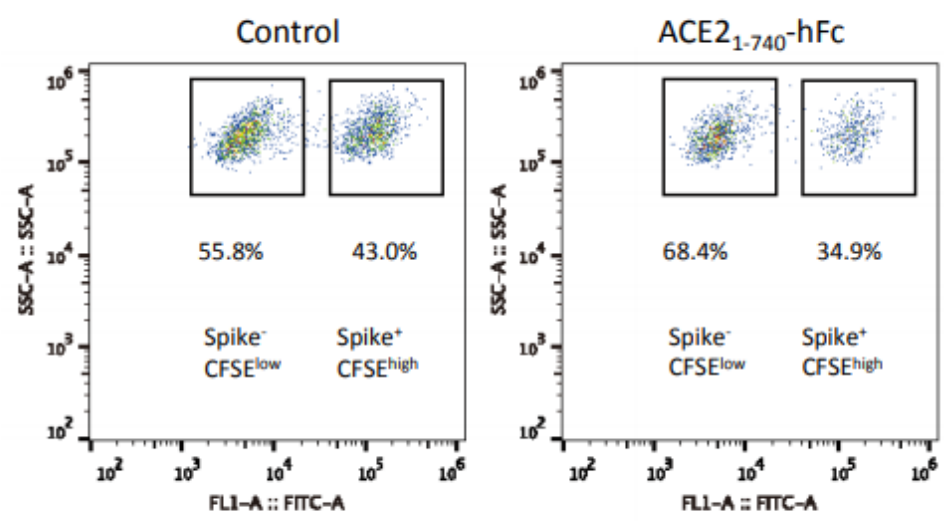

B
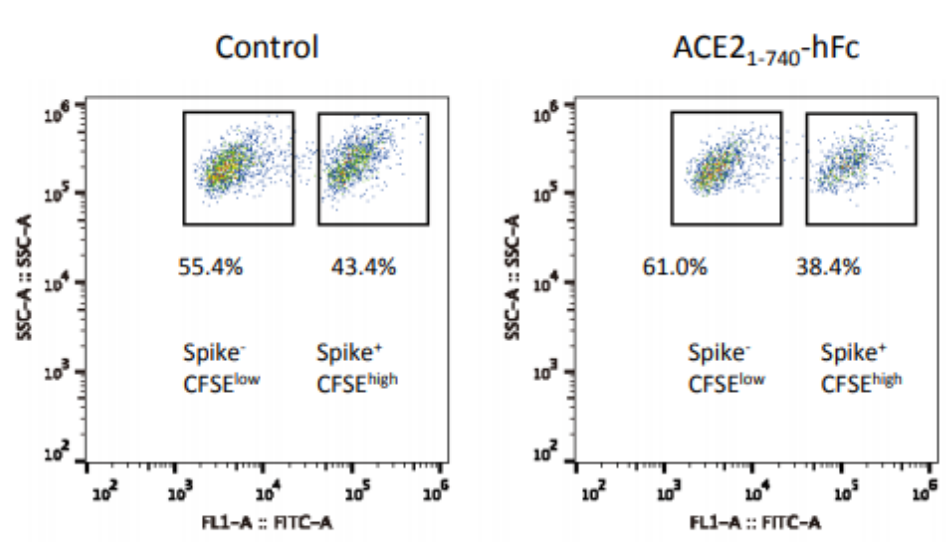
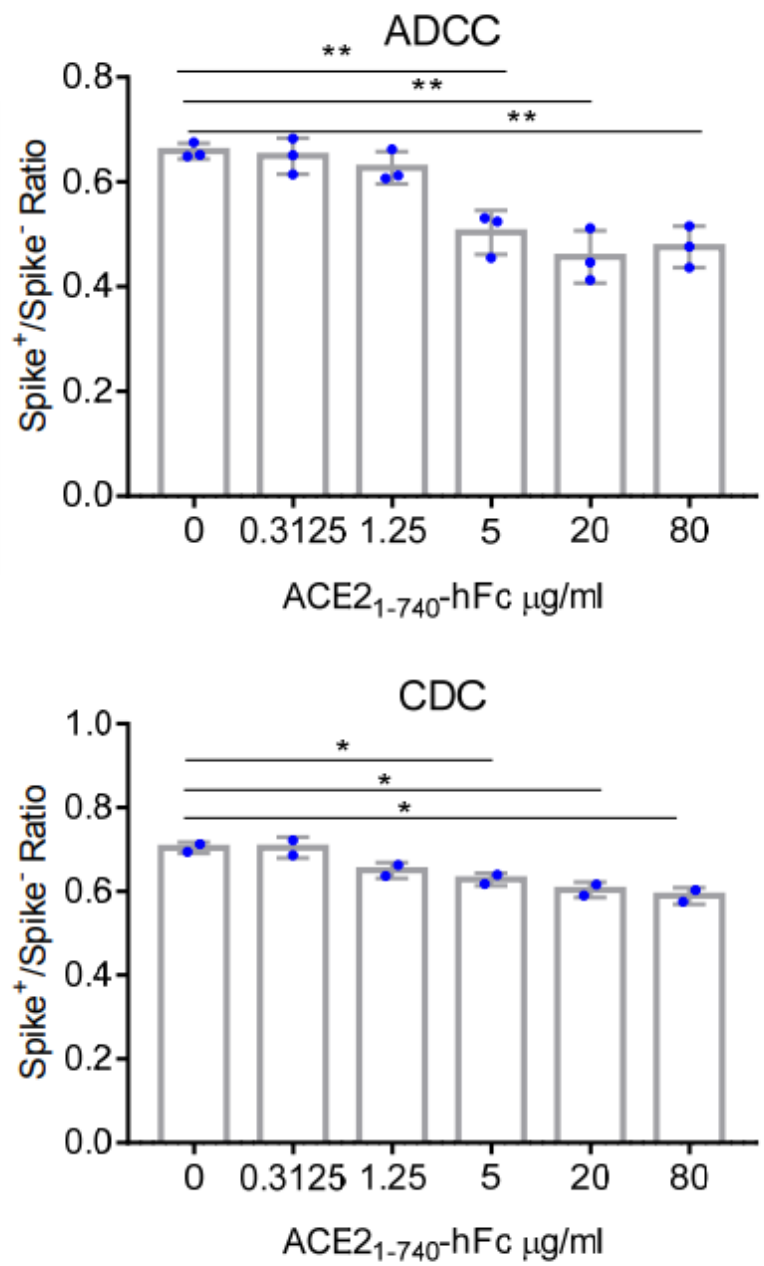

Figure 3

ACE21-740-hFc induced SARS-CoV-2 spike+ cell death through ADCC and CDC; 
C
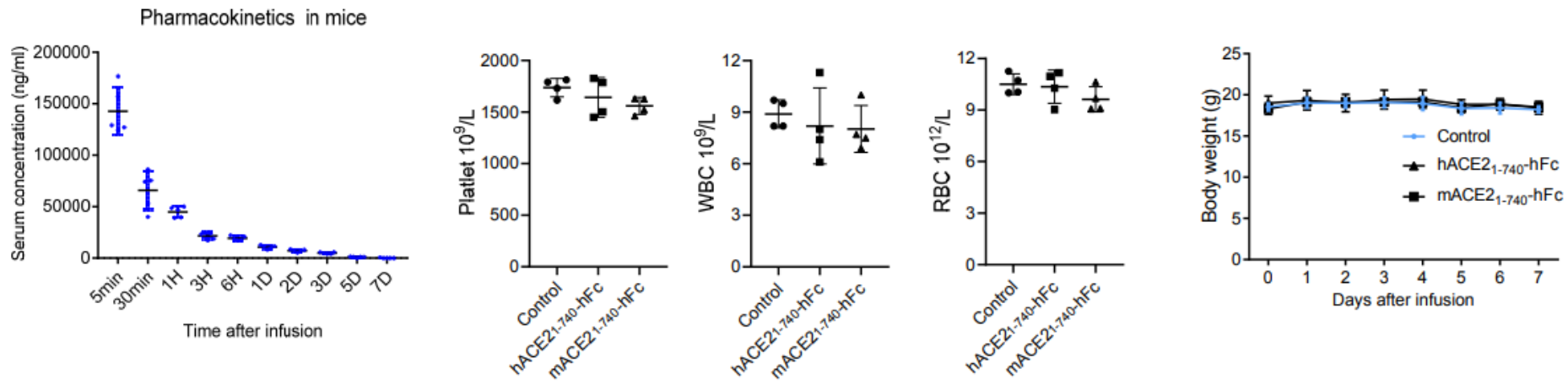

D

E
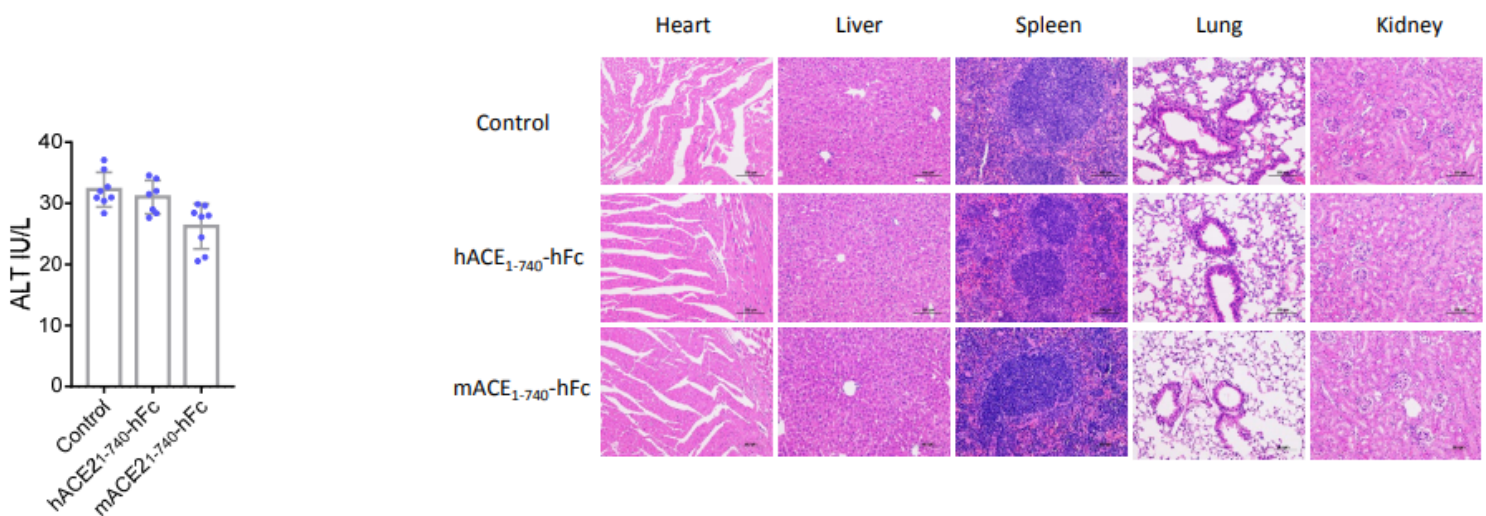

Figure 4

Safety profile of ACE21-740-hFc in mice. 
A

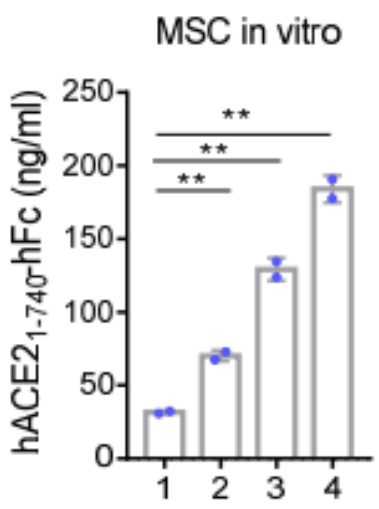

Days after culture

C

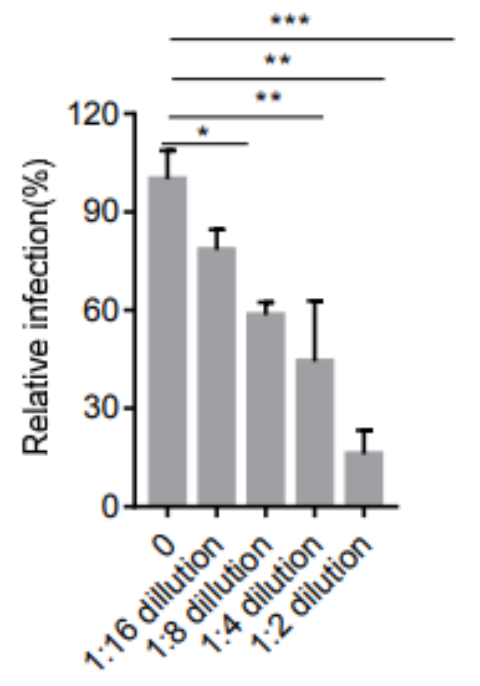

MSC-ACE2 ${ }_{1-740}-\mathrm{hFC}$ supernatant
B

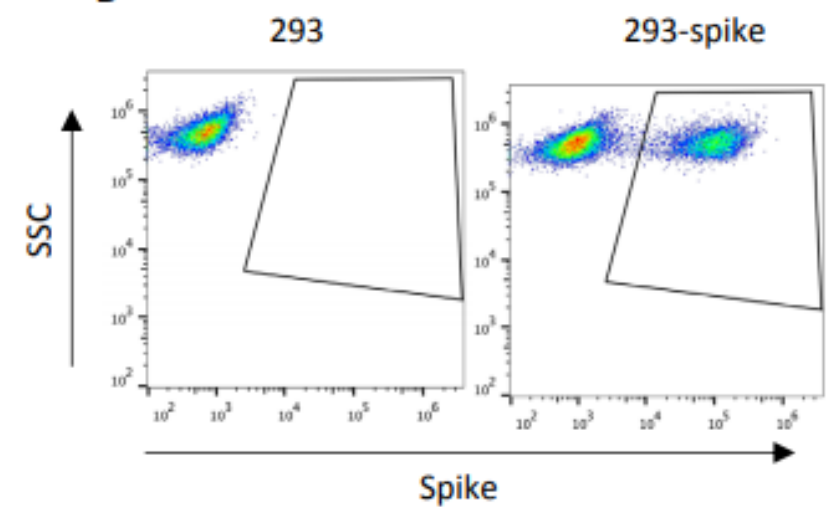

$E$

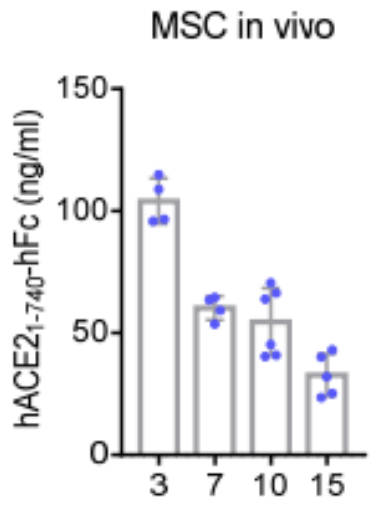

Days after infusion

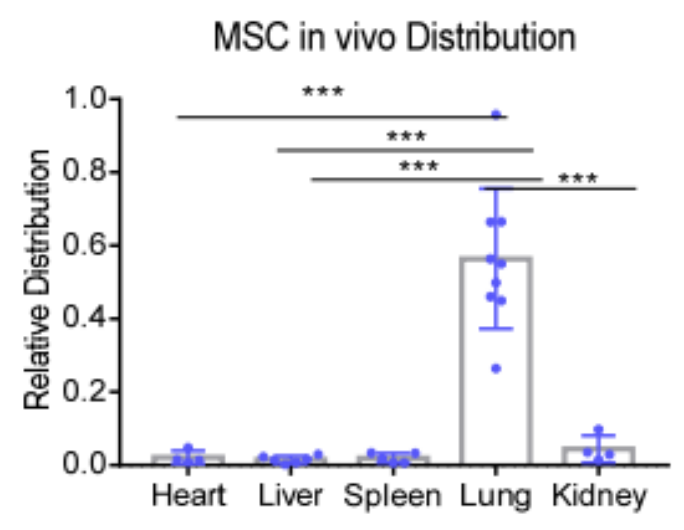

Figure 5

Characterization of engineered MSCs expressing ACE21-740-hFc. 
TF-1 proliferation assay

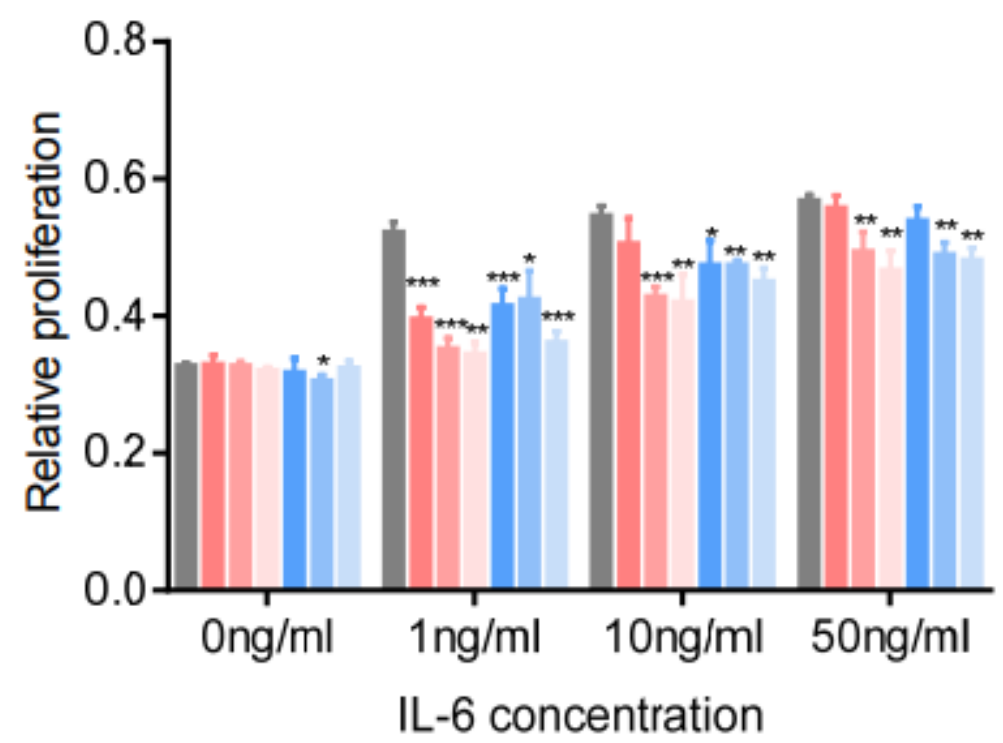

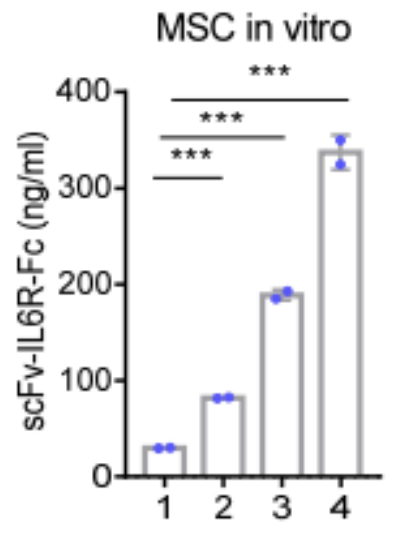

Days after culture
C

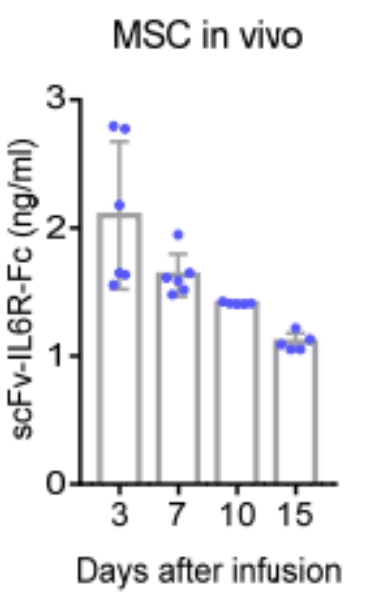

Figure 6

Characterization of engineered MSCs expressing scFv-IL6R-Fc.

\section{Supplementary Files}

This is a list of supplementary files associated with this preprint. Click to download.

- Supplemenalinformation.pdf 